\title{
The Light Side and The Dark Side of the Milky Way Halo
}

\author{
Prajwal R. Kafle ${ }^{1}$, Sanjib Sharma ${ }^{1}$, Geraint F. Lewis ${ }^{1}$ and Joss \\ Bland-Hawthorn ${ }^{1}$ \\ ${ }^{1}$ Sydney Institute for Astronomy, School of Physics, The University of Sydney \\ email: p.kafle@physics.usyd.edu.au
}

\begin{abstract}
We present our recent measurement of the kinematics of the Milky Way stellar halo (Light Side) and the derived mass of the dark matter halo (Dark Side) using the Jeans analysis. A tangential dip in the velocity anisotropy profile at $r \sim 17 \mathrm{kpc}$ (Kafle et al. 2012), and a distinct difference of $\sim 65 \mathrm{kms}^{-1}$ in the mean azimuthal velocity and the r.m.s dispersion of the most metal-rich and the metal-poor Blue Horizontal Branch stars we find (Kafle et al. 2013) are reported. The implications of this on the current controversial issue of an existence of the two-components in the halo are also discussed.

Aided with the kinematic measurements of the light side, we demonstrate how we infer the dynamical property of the dark side. Considering a realistic three component galaxy model (Hernquist bulge, Miyamoto-Nagai disk and NFW halo), we estimate the virial mass of the Galaxy to be $M_{\mathrm{vir}}=1.2_{-0.4}^{+0.5} \times 10^{12} M_{\odot}$ (Kafle et al. 2012 ). We also show that the rotation curve of the Galaxy has undulations similar to what have also been seen in the studies of the HI gas (Sofue et al. 2009).
\end{abstract}

Keywords. Galaxy:halo, Galaxy:kinematics and dynamics, stars:horizontal-branch
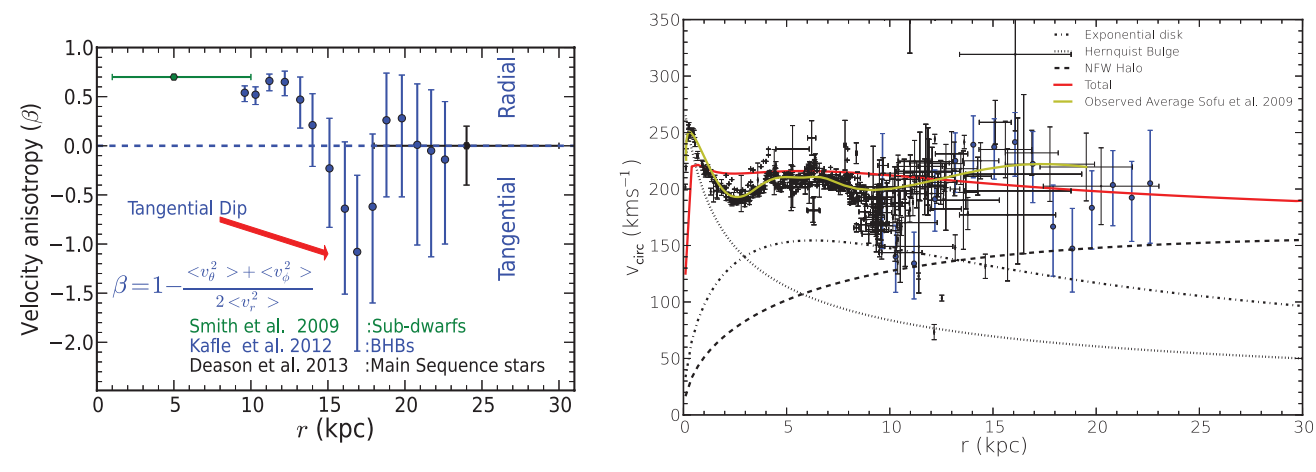

Figure 1. The anisotropy (left) and circular velocity (right) profile of the halo along the galacto-centric distance $r$. Blue dots with error bars are our measurements whereas other markers with error bars are estimates taken from the literature for the various classes of the stellar population.

\section{References}

Sofue, Y., Honma, M., Omodoka, T. 2009, PASJ, 61, 227

Kafle, P. R., Sharma, S., Lewis, G. F., \& Bland-Hawthorn, J. 2012, ApJ, 761, 98

Kafle, P. R., Sharma, S., Lewis, G. F., \& Bland-Hawthorn, J. 2013, MNRAS, 430, 2973-2978 